\title{
Permanent Data
}

National Cancer Institute

\section{Source}

National Cancer Institute. Permanent Data. NCI Thesaurus. Code C142634.

Data that is not, under normal circumstances, subject to change. 